Chirurg 2014 $\cdot 85: 822$

DOI 10.1007/s00104-014-2856-1

Online publiziert: 17. August 2014

๑) Springer-Verlag Berlin Heidelberg 2014
K. Götzky · J. Jähne

Klinik für Allgemein- und Viszeralchirurgie, Schwerpunkt für endokrine und onkologische Chirurgie,

Diakoniekrankenhaus Henriettenstiftung Hannover

\title{
Lebensqualität nach Operation bei Barrett-Frühkarzinom
}

\section{Ein prospektiver Vergleich zwischen Ivor-Lewis- und modifizierter Merendino-Resektion}

Nach Ausschluss (Tod, Tumorrezidiv) konnten 60 Fälle in der TER- und 27 Fälle in der MER-Gruppe ausgewertet werden. Die Rücklaufquote für den validierten Fragebogen der European Organisation for Research and Treatment of Cancer betrug $89 \%$ nach 1 bzw. $87 \%$ nach 2 Jahren. Als Kontrollgruppe fungierten 67 Patienten mit neudiagnostiziertem eACE.

\section{Hintergrund}

Aufgrund einer regelmäßigen endoskopischen Überwachung von Refluxpatienten gelingt es zunehmend, ein Barrett-Frühkarzinom (eACE) zu diagnostizieren. Als Alternative zur transthorakalen Ösophagusresektion (TER) wird die Merendino-Resektion (MER) eingesetzt, von der postuliert wird, zum einen weniger Morbidität (TER 30-40\%) und Mortalität (TER bis 5\%) auszulösen, zum anderen eine bessere postoperative Lebensqualität (QL) zu ermöglichen. Die vorliegende Studie soll mittels Fragebögen die QL 1 bzw. 2 Jahre nach beiden Operationen evaluieren und vergleichen.

\section{Material und Methoden}

Zwischen Juli 2000 und Juli 2007 wurden in der Horst-Schmidt-Klinik Wiesbaden 117 Patienten mit Barrett-Frühkarzinom (uT1) operiert. Patienten ohne Voroperation, mit einem endoskopisch kurzen Barrett-Segment und geringem chirurgischem Risikoprofil wurden dabei der MER zugeteilt. Die TER und die vaguserhaltende MER erfolgten standardisiert.

\section{Ergebnisse}

Die Patientengruppen TER und MER wiesen keine signifikanten Unterschiede in Alter, Geschlecht, Body-Mass-Index, ASA und Tumorstadium auf. Operationsdauer, Krankenhausaufenthalt, Komplikations- und Reoperationsrate waren vergleichbar.

Verglichen mit der Kontrollgruppe gab es in der MER 1 und 2 Jahre nach Operation mehr postoperative Beschwerden als bei Patienten der TER. Verglich man TER und MER 1 Jahr nach der Operation, gab es mehr Beschwerden nach MER (Übelkeit/Erbrechen, Appetitminderung, Schmerzen) als nach TER (Dysphagie, Verstopfung). Zudem hatten MER-Patienten anfangs noch emotionale und soziale Schwierigkeiten. Nach 2 Jahren waren kaum noch Unterschiede festzustellen, allerdings gaben TER-Patienten weiter Dysphagie an.

\section{Diskussion}

Diese Studie untersuchte als Erste die Lebensqualität nach modifizierter MER und TER. Die angegebenen Beeinträchtigungen traten bei weniger als $50 \%$ der Patienten auf und zeigten verglichen mit der Kontrollgruppe in 80\% keine signifikanten Unterschiede. Daher konnte festgestellt werden, dass sich Patienten nach Ösophagusresektion gemäß Maßstab des Fragebogens komplett erholen. In der frühpostoperativen Phase gab es mehr Beeinträchtigung bei MER, sodass diese Operation nicht überlegen ist. Da nach 2 Jahren zwischen TER und MER insgesamt keine wesentlichen Unterschiede feststellbar waren, scheinen beide Methoden im Hinblick auf die QL vergleichbar.

Angemerkt werden muss, dass zum einen die Patienten aus der Kontrollgruppe bez. ihrer Merkmale nicht mit den übrigen Patienten verglichen wurden und dass es sich nicht um eine randomisierte Studie handelte, sondern die Patienten für die MER ausgewählt wurden. Somit sollten die Ergebnisse in weiteren Studien gesichert werden.

\section{Korrespondenzadresse}

\section{Dr. K. Götzky}

Klinik für Allgemein- und Viszeralchirurgie, Schwerpunkt für endokrine und onkologische Chirurgie, Diakoniekrankenhaus Henriettenstiftung Hannover, Marienstr. 72-90, 30171 Hannover kristina.goetzky@ddh-gruppe.de

Interessenkonflikt. K. Götzky und J. Jähne geben an, dass kein Interessenkonflikt besteht. 